\title{
Calculation of Bleeding Assessment Tool Score in all Patients of Bleeding Diathesis and its Correlation with Factor Assays: Experience in a Tertiary Care Centre
}

Dr. Vibha Gupta ${ }^{1}$, Dr. Mansi Kala ${ }^{1 *}$, Dr. Kunal Das ${ }^{2}$, Dr. Vikas Shrivastava ${ }^{1}$, Dr. Anuradha Kusum ${ }^{3}$, Dr. Dushyant Singh Gaur $^{3}$, Dr. Meena Harsh ${ }^{3}$, Dr. Neena Chauhan ${ }^{3}$, Dr. Nadia Shirazi ${ }^{3}$, Dr. Smita Chandra ${ }^{3}$, Dr. Sushil Shukla ${ }^{4}$, Dr. Neetika Agarwal $^{1}$, Dr. Meghali Debane ${ }^{1}$

${ }^{1}$ Assistant Professor, Department of Pathology, HIHT Medical College, Dehradun

${ }^{2}$ Assistant Professor, Department of Paediatrics, HIHT Medical College, Dehradun

${ }^{3}$ Professor, Department of Pathology, HIHT Medical College, Dehradun

${ }^{4}$ Senior Resident, Department of Pathology, HIHT Medical College, Dehradun

DOI: $10.36348 /$ sjpm.2020.v05i01.003 $\quad$ | Received: 03.01.2020 | Accepted: $19.01 .2020 \mid$ Published: 28.01 .2020

*Corresponding author: Dr. Mansi Kala

\section{Abstract}

Background: The congenital bleeding disorders haemophilia A and B are estimated to affect between one in 10000 and one in 50000 males. The clinical profile of hemophilia patients is rarely reported in India. ISTH- Bleeding Assesment Tool score is one such score which not only helps us to diagnose a bleeding diathesis but may also help us in predicting the severity of bleeding potential of any patient with bleeding diathesis. Objective: To describe the clinical and epidemiological profile of hemophiliac patients in Uttarakhand. Calculate Bleeding Assessment Tool score in all patients of haemophilia and differentiate severe haemophilia from moderate haemophilia. Material and Methods: A total of 75 patients of all age groups with haemophilia A and B were included in the study. A detailed clinical history using ISTH BAT along with clinical assessment by the hematologist was done. Special emphasis was laid on demographic details. Coagulation studies were done on STAGOSTA compact. Chi-square test was used for Assessing BAT scores. Results: The hospital based prevalence was 1.2\% for haemophilia A and $0.3 \%$ for haemophilia B in our study. There were $74 \%$ severe haemophilia and 5\% of mild haemophilia in our study. The presence of inhibitors was $6 \%$. There was significant statistical difference between the BAT score values of moderate and severe haemophilia ( $\mathrm{p}$ value $<0.05)$. Conclusion: The study suggested high prevalence of haemophilia in the state along with high numbers of inhibitors. More studies are required to establish the role of bleeding assessment tool in differentiating moderate haemophilia from severe haemophilia.

Keywords: Bleeding Assesment Tool Score, Coagulation studies, Hemophilia.

Copyright @ 2020: This is an open-access article distributed under the terms of the Creative Commons Attribution license which permits unrestricted use, distribution, and reproduction in any medium for non-commercial use (NonCommercial, or CC-BY-NC) provided the original author and sources are credited.

\section{INTRODUCTION}

Inherited bleeding disorders (IBDs) are caused by quantitative and qualitative alterations of either platelets or plasma proteins involved in coagulation and fibrinolysis. Hemophilias are the most frequent IBD. The congenital bleeding disorders haemophilia A and B are estimated to affect between one in 10000 and one in 50000 males [1]. Studies of these diseases revealed that they result in varying degrees of bleeding diathesis. This deserves attention, not only to quantitative abnormalities but also to some IBDs, which reflect the synthesis of dysfunctional coagulation proteins or production of abnormal platelets [2]. Hemophilias are the most frequent IBDs. However, von Willebrand disease (VWD) and platelet function defects (PFDs) are less common causes of bleeding. Various studies have reported that VWD is the most common congenital bleeding disorder in the population [1, 3, 4]. Hemophilia is an inherited bleeding disorder caused by deficiency of coagulation factor VIII (hemophilia A), factor IX (hemophilia B). They are X-linked recessive disorder [5]. Hemophilia $\mathrm{A}$ is more common than hemophilia B. Hemophilia A is also more likely to be severe. Hemophilias are characterized as mild, moderate, or severe, based on the residual or baseline factor activity level expressed as a percent of normal or in international units (IU)/ML [6]. Factor levels typically correlate with the degree of bleeding symptoms. Severe hemophilia - defined as $<1 \%$ factor activity, moderate hemophilia - defined as a factor activity level $\geq 1$ and $\leq 5 \%$ factor activity and mild 
hemophilia is defined as a factor activity level $>5$ and $<40 \%$ of factor activity [7]. Most common symptoms of hemophilia are spontaneous bleeding, hematomas, hemarthrosis, hematuria, soft tissue bleeding including life threatening bleeds of the CNS, oral bleeds etc. Frequency depends on severity and genotype [8]. Common sites of bleeding in newborns include the central nervous system, extracranial sites such as cephalohematoma, and sites of medical interventions including circumcision, heel sticks, and venipunctures [9]. Bruising, joint bleeds, and other sites of musculoskeletal bleeding become more common once children begin walking. Common sites of bleeding in older children and adults include joints and muscles [10]. The overall frequency of bleeding has declined with greater use of prophylactic factor administration. It is estimated that $10-80 \%$ of people with hemophilia are present in developing countries such as India [11]. However, majority of them remain under-diagnosed or not registered. Given that the incidence of hemophilia A is one in 5,000 and hemophilia B is one in 30,000, as in the US, one expects India to have close to 100,000 cases of haemophilia.However, according to the World Federation of Hemophilia, with data provided by the Hemophilia Federation of India (HFI), only 13,448 patients are registered [12]. The clinical profile of hemophilia patients is rarely reported in India. Only hospital-based studies have estimated the burden of hemophilia because the epidemiological methods are not cost-effective for the measurement of this rare diseases. Thus, registries remain the best source for obtaining the epidemiological data on these conditions [13]. Although the genetic basis of this disorder has been well studied in India, data regarding the number of patients, disease trends, demographic profile, costs incurred through a public health program have not been reported [14]. To suspect the bleeding disorder, a history of mucocutaneous bleeding is an important component. ISTH- Bleeding Assesment Tools score is one such score which not only helps us to diagnose a bleeding diathesis but may also help us in predicting the severity of bleeding potential of any patient with bleeding diathesis [15]. The objective of this study was to describe the clinical and epidemiological profile of hemophiliac patients in north India particularly in Uttarakhand. To calculate Bleeding Assessment Tool score in all patients of haemophilia and differentiate severe haemophilia from moderate haemophilia.

\section{MATERIAL AND METHODS}

An observational cross-sectional study was conducted in the department of Pathology, Himalayan Institute of Medical Sciences, Dehradun, Uttarakhand, India. The study was during 2017-18 and approved by the ethical committee of the institute. The consent was taken from each participant's parent/guardian before enrolling in the study. The objective of the study was explained to the parent /guardian and confidentiality of identity was assured. A total of 75 patients of all age groups with haemophilia A and B were included in the study. All the patients included were known cases of haemophilia A and B and cases of other bleeding diathesis were excluded from the study. A detailed clinical history was elicited from the accompanying parent/guardian using ISTH -BAT along with clinical assessment by the haematologist. Special emphasis was laid on demographic details. Coagulation studies were done on STAGO STA Compact. End point detection of clot was done using electromechanical method. Factor assay was done by “one-stage assay using factor VIII deficient plasma, Factor IX deficient plasma, calibrate and controls. Factor level of $(<1 \%),(1-4 \%)$, and (5$40 \%)$ defined as severe, moderate, and mild hemophilia, respectively. Inhibitor screen was done in all cases. Confirmation and quantification of inhibitors was done with Bethesda assay (B.U). The quality control for the instrument (STAGO) used for coagulation study were taken care as per the NABL guidelines. Data was collected and entered in M.S. Excel 2010. The different statistical analysis was performed using SPSS software version 22. The one sample Kolmogorov-Smirnov test were employed to determine whether the data sets were normally distributed or not. Normally distributed data was analysed using parametric test and non normally distributed data was analysed using non parametric test. Descriptive statistics was calculated for quantitative variables. Frequency along with percentages were calculated for qualitative and categorical variables. Graphical representation of the variables are shown to understand the results clearly. Chi-square test was used to find the statistical difference between the BAT score values of severe hemophilia and moderate hemophilia.

\section{RESULTS}

This cross sectional study was carried out to study the demographic, clinical profile, laboratory assays and inhibitor profile of patients with Hemophilia $\mathrm{A}$ and B. We drew the following results from this study. We found $82.6 \%(62 / 75)$ of our patients belonged to haemophilia A, while $17.3 \%$ (13/75) were Haemophilia B. Of all the patients, $56 \%$ gave a positive family history of haemophilia. Figure 1, shows the age wise distribution of patients with hemophilia with maximum patients in the age group of 11-20 years and eighteen patients in less than 10 years age group. Severity wise distribution of Haemophilia A and Haemophilia B patients showed (Fig-2) 72\% (54/75) patients were of severe haemophilia and only $4 \%(3 / 75)$ were mild haemophilia. Maximum patients of haemophilia belonged to the capital city of Uttarakhand (Table-1). The distribution of mild moderate and severe haemophilia with their resoective BAT score suggested that most of the patients of severe haemophilia had BAT score above 11 . There was only one case of severe haemophilia which had an unusually low BAT score indicating phenotypic heterogeneity. Most cases of moderate haemophilia had a BAT score of 3-10 (Figure-3). More than 50 days of factor exposure was seen in only $13 \%$ of our population. Most common 
clinical feature of haemophilia was cutaneous bleeds and 5 patients of haemophilia had CNS bleeds (Figure4). Most common first clinical presentation was also cutaneous bleeds in our patients. There were four cases of inhibitors in our study and all of them had an unusually high Bethesda Score (Table-2).

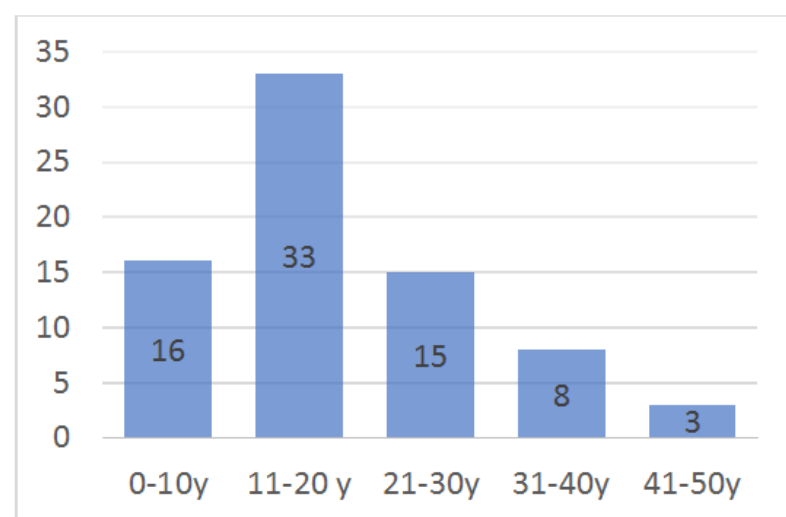

Fig-1: Age wise distribution of patients with Hemophilia

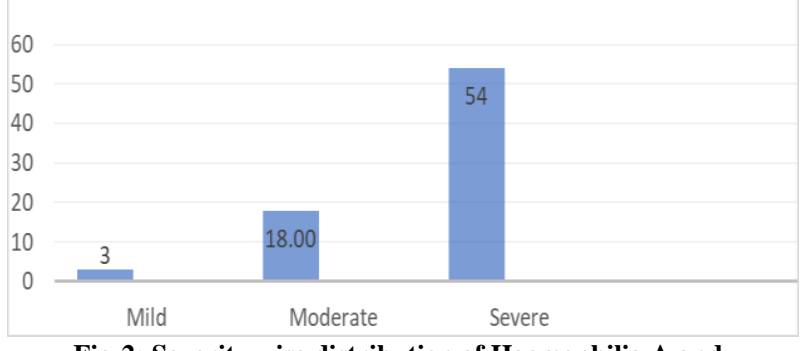

Fig-2: Severity wise distribution of Haemophilia A and Haemophilia B patients

Table-1: District wise distribution of patients of Hemophilia in the state of Uttarakhand

\begin{tabular}{|l|l|l|}
\hline S. No & District & Number of patients \\
\hline 1. & Dehradun & 33 \\
\hline 2. & Haridwar & 21 \\
\hline 3. & Pithoragarh & 04 \\
\hline 4. & Kotdwar & 04 \\
\hline 5. & Nainital & 04 \\
\hline 6. & Rishikesh & 03 \\
\hline 7. & Udham Singh Nagar & 02 \\
\hline 8. & Almoda & 02 \\
\hline 9. & Tanda & 02 \\
\hline
\end{tabular}

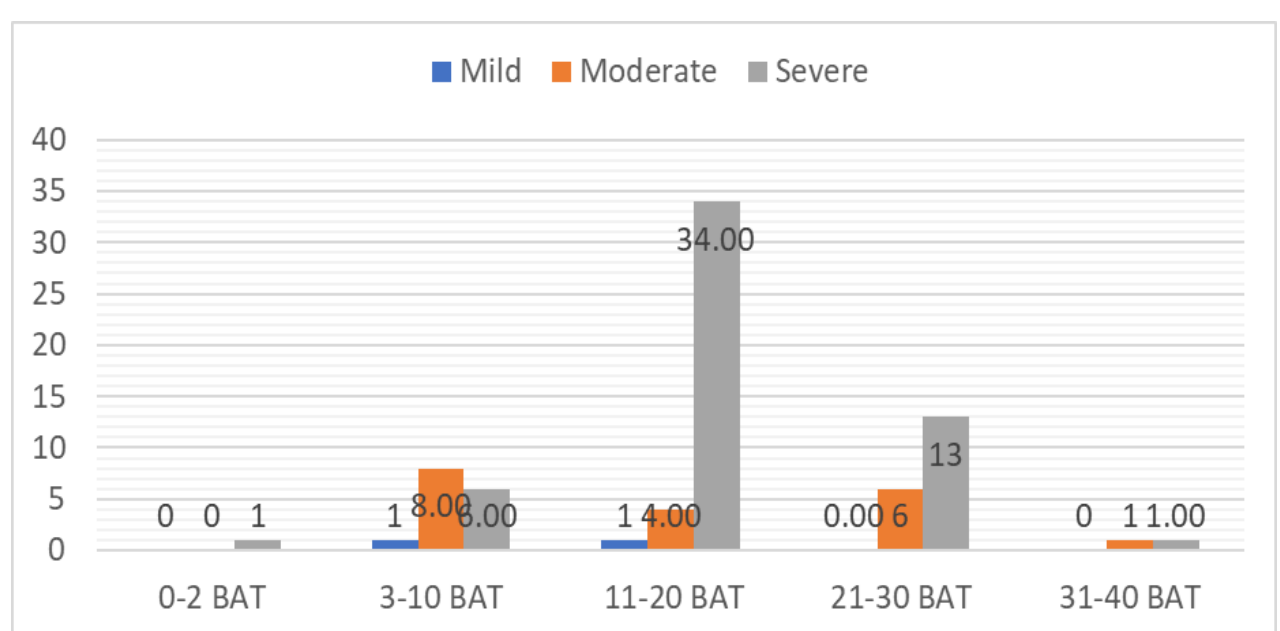

Fig-3: Distribution of mild, moderate and severe cases of Hemophilia and respective BAT scores

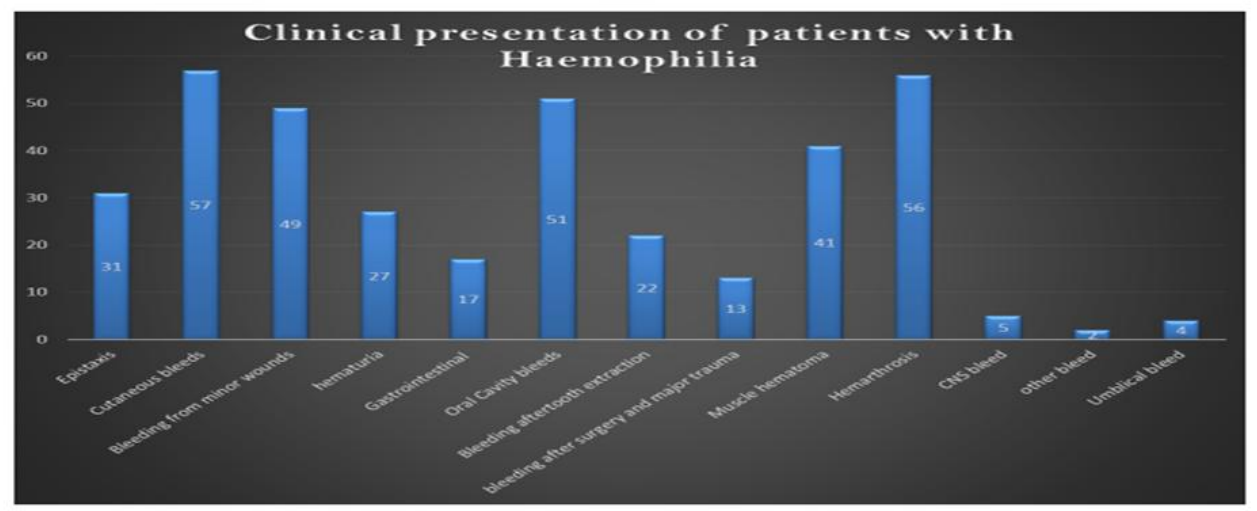

Fig-4: Clinical presentation of patients with haemophilia 
Table-2: Patients of Hemophilia A with inhibitors

\begin{tabular}{|r|r|r|r|r|r|r|}
\hline & AGE & BAT SCORE & A/B & GRADE & $\begin{array}{r}\text { INHIBITOR } \\
\text { SCREEN }\end{array}$ & BETHESDA \\
\hline 1. & 34 & 24 & A & SEVERE & POSITIVE & 70.4 B.U \\
\hline 2. & 42 & 19 & A & SEVERE & POSITIVE & 70.4 B.U \\
\hline 3. & 18 & 24 & A & SEVERE & POSITIVE & 614 B.U \\
\hline 4. & 26 & 16 & A & SEVERE & POSITIVE & 28 B.U \\
\hline
\end{tabular}

\section{DISCUSSION}

According to WFH data 2016 estimated prevalence of haemophilia A in India and USA is 1.1 and $4 \%$ respectively whereas for haemophilia $\mathrm{B}$ is 0.17 and 1.2 percent respectively [16].

The hospital based prevalence derived from our data was $1.2 \%$ for haemophilia $\mathrm{A}$ and $0.3 \%$ for haemophilia B, although this estimate could not be extrapolated to the whole state, still our data stood close to national prevalence rate for haemophilia $\mathrm{A}$. These variations in prevalence rates between states and countries could be due to geographic and racial differences, variations in case finding, haemophilia awareness, public health services, and dissimilar diagnostic methodologies due to a lack of standardized laboratory testing.

Being an inherited $\mathrm{X}$ linked disorder, family history has been observed in $40-71 \%$ cases of haemophilia, however in our study, it was positive in $44 \%$ of the cases $[17,18]$. Most of the patients were residing in big cities of Uttarakhand like Dehradun, Kotdwar and Haldwani. Factor availability in only big cities of Uttarakhand, was probably the main reason behind this skewed distribution of patient population.

We found $74 \%$ severe haemophilia and $5 \%$ of mild haemophilia in our study. According to WFH 2016 data detection of mild haemophilia in high income groups is as high as $34 \%$, suggesting that, we may be missing the mild haemophilia cases [16]. Also severe haemophilia is reported to be most prevalent with its proportion ranging from $43 \%$ to $55.7 \%$ according to many studies [19-21]. Most common complaints in our patients was cutaneous bleeds with a substantial number of patients having joint problems. Nearly $75 \%$ of the patients in our study developed joint disability. Studies done by Kar A et al., and Kempton $\mathrm{Cl}$ et al., has shown joint disability to be as high as $92 \%$ and $53 \%$ respectively in their patient population $[15,23]$. This highlights the crippling nature of this disease, and our state was no different. The first clinical manifestation is reported to be post traumatic bleeds, however in our study spontaneous cutaneous bleeds were most common.
Factor exposure was $77 \%$ in our patient population and prophylactic therapies were being given to only to a few patients. Most of the patients were given factor replacement in case of surgery or trauma. The presence of inhibitors in our hospital based study was $6 \%$ of the total patients. They were all high titre inhibitors. Development of inhibitors in India has been reported to be as high as $8.3-13 \%$ by Gosh $\mathrm{K}$ et al., [24] Some studies confirms the importance risk factors like young age at exposure, race, and a family history of inhibitors [25-27] however our study was unable to establish any of these risk factors because the data with regard to inhibitors was less. Also our study showed unusually high levels of B.U in all the positive cases of inhibitors and we did not get any low titre inhibitor, this was in contrast to the previous studies which had shown a good percentage of low titre inhibitors as well [27]. Our maximum patients were in the age group of 11-20 years, whereas data from developed countries showed almost equal distribution in all the age groups [16]. Such a skewed age group in our study may suggest the high mortality rate in this these patients. Phenotypic heterogeneity is a known phenomenon in haemophilia where severe haemophiliacs also behave like moderate or mild haemophiliacs, this is because some cases despite having low Factor levels have good thrombin generation, which reduces the patients need for factor replacement [28]. We encountered one case of phenotypic heterogeneity where a patient of severe haemophilia had not required factor replacements. Studies have shown phenotypic heterogeneity to be as high as $10-15 \%$ in some patient populations $[28,29]$.

Most cases of severe Haemophilia had a Bleeding Assessment Tool score [15] between 11-20 whereas most cases of moderate haemophilia the score ranged from 3-10. Cases of mild haemophilia were very few to comment upon. There was significant statistical difference between the BAT score values of moderate and severe haemophilia ( $\mathrm{p}$ value $<0.05$ ). Differentiating moderate haemophilia from severe haemophilia is important because moderate haemophilia seldom experiences unprovoked bleeding and have much better function of joint functions, therefore prophylaxis may not be needed in them. More studies are required to 
establish whether BAT score can help us in triaging patients of haemophilia for prophylactic therapy.

\section{CONCLUSION}

This study aimed to study the epidemiological profile of patients with haemophilia. The hospital based surveys the possibility high prevalence of haemophilia in the state along with high numbers of inhibitors. More studies are required to establish the role of Bleeding assessment tool in differentiating moderate haemophilia from severe haemophilia. The inferred high mortality rate in haemophilia is worrisome in the state. Low numbers of mild haemophilia suggests hidden cases in the state. More studies are required to study about inhibitor levels and factors influencing them. Awareness is important so that these genetic diseases could be picked up using simple questionnaires such as BAT scores, especially in interiors of Uttarakhand.

\section{REFERENCES}

1. Blanchette, V. S., Sparling, C., \& Turner, C. (1991). 2 Inherited bleeding disorders. Bailliere's clinical haematology, 4(2), 291-332.

2. Lippi, G., Franchini, M., \& Guidi, G. C. (2007). Diagnostic approach to inherited bleeding disorders. Clinical Chemical Laboratory Medicine, 45(1), 2-12.

3. Ziv, O., \& Ragni, M. V. (2004). Bleeding manifestations in males with von Willebrand disease. Haemophilia, 10(2), 162-168.

4. Federici, A. B. (2006, September). Diagnosis of inherited von Willebrand disease: a clinical perspective. In Seminars in thrombosis and hemostasis, 32(6), 555-565.

5. Srivastava, A., Tariq, M., \& Heijnen, L. (2001). Haemophilia in Developing Countries - the challenge of detection and diagnosis. Comprehensive Haemophilia Care in Developing Countries. Lahore, Pakistan: Feroze sons, 17-25.

6. Blanchette, V. S., Key, N. S., Ljung, L. R., Manco-Johnson, M. J., Van Den Berg, H. M., Srivastava, A., \& Subcommittee on Factor VIII, Factor IX and Rare Coagulation Disorders. (2014). Definitions in hemophilia: communication from the SSC of the ISTH. Journal of Thrombosis and Haemostasis, 12(11), 1935-1939.

7. White, G. (2001). Factor VIII and Factor IX Subcommittee. Definitions in hemophilia. Recommendation of the scientific subcommittee on factor VIII and factor IX of the scientific and standardization committee of the International Society on Thrombosis and Haemostasis. Thromb Haemost., 85, 560.

8. Venkateswaran, L., Wilimas, J. A., Jones, D. J., \& Nuss, R. (1998). Mild hemophilia in children: prevalence, complications, and treatment. Journal of pediatric hematology/oncology, 20(1), 32-35.

9. Kulkarni, R., Presley, R. J., Lusher, J. M., Shapiro, A. D., Gill, J. C., Manco-Johnson, M., ... \&
Mathew, P. (2017). Complications of haemophilia in babies (first two years of life): a report from the Centers for Disease Control and Prevention Universal Data Collection System. Haemophilia, 23(2), 207-214.

10. Kulkarni, R., Soucie, J. M., Lusher, J., Presley, R., Shapiro, A., Gill, J., ... \& Dimichele, D. (2009). Sites of initial bleeding episodes, mode of delivery and age of diagnosis in babies with haemophilia diagnosed before the age of 2 years: a report from The Centers for Disease Control and Prevention's (CDC) Universal Data Collection (UDC) project. Haemophilia, 15(6), 1281-1290.

11. Mammen, J., Nair, S. C., \& Srivastava, A. (2007, April). External quality assessment scheme for hemostasis in India. In Seminars in thrombosis and hemostasis, 33(3):265-272.

12. Report on the annual global survey 2008. Montreal: World Federation of Hemophilia; 2009.

13. Dalal, A., Pradhan, M., \& Agarwal, S. (2006). Genetics of bleeding disorders. International journal of human genetics, 6(1), 27-32.

14. Kar, A., Phadnis, S., Dharmarajan, S., \& Nakade, J. (2014). Epidemiology \& social costs of haemophilia in India. The Indian journal of medical research, 140(1), 19.

15. Elbatarny, M., Mollah, S., Grabell, J., Bae, S., Deforest, M., Tuttle, A., ... \& Riddel, J. (2014). Normal range of bleeding scores for the ISTHBAT: adult and pediatric data from the merging project. Haemophilia, 20(6), 831-835.

16. World Federation of Hemophilia (WFH) Canada: WFH; 2016. [accessed on May 9, 2016]. Report on the Annual Global Survey 2011. Available from: http://www1.wfh.org/publications/files/pdf1488.pdf

17. Kar, A., \& Potnis-Lele, M. (2001). Descriptive epidemiology of haemophilia in Maharashtra, India. Haemophilia, 7(6), 561-567.

18. Karim, M. A., Siddique, R., Jamal, C. Y., \& Islam, A. (2013). Clinical profile of haemophilia in children in a tertiary care hospital. Bangladesh Journal of Child Health, 37(2), 90-96.

19. Soucie, J. M., Evatt, B., Jackson, D., \& Hemophilia Surveillance System Project Investigators. (1998). Occurrence of hemophilia in the United States. American journal of hematology, 59(4), 288-294.

20. Hazewinkel, M. H., Hoogerwerf, J. J., Hesseling, P. B., Hartley, P., MacLean, P. E., Peters, M., \& Wessels, G. (2003). Haemophilia patients aged 0 18 years in the Western Cape. South African Medical Journal, 93(10), 793-796.

21. Rodgers, M. G., \& Greenburg, S. C. (1998). Wintrobe's Clinical Haematology. 10th ed. Baltimore: Wilkins; Inherited coagulation disorders; 1683-732.

22. Kim, K., \& Cho, M. J. (1988). Comprehensive Clinical and Statistical Analysis of 
Hemophilia. Journal of Korean medical science, 3(3), P107-115.

23. Kempton, C. L., Recht, M., Neff, A., Wang, M., Buckner, T. W., Soni, A., ... \& Cooper, D. L. (2018). Impact of pain and functional impairment in US adults with haemophilia: Patient-reported outcomes and musculoskeletal evaluation in the pain, functional impairment and quality of life ( $\mathrm{P}$ FiQ) study. Haemophilia, 24(2), 261-270.

24. Ghosh, K., Shetty, S., Kulkarni, B., Nair, S., Pawar, A., Khare, A., ... \& Mohanty, D. (2001). Development of inhibitors in patients with haemophilia from India. Haemophilia, 7(3), 273278.

25. Lusher, J., Abildgaard, C., Arkin, S., Mannucci, P. M., Zimmermann, R., Schwartz, L., \& Hurst, D. (2004). Human recombinant DNA-derived antihemophilic factor in the treatment of previously untreated patients with hemophilia A: final report on a hallmark clinical investigation. Journal of Thrombosis and Haemostasis, 2(4), 574-583.

26. Chalmers, E. A., Brown, S. A., Keeling, D., Liesner, R., Richards, M., Stirling, D., ... \& Paediatric Working Party of UKHCDO. (2007). Early factor VIII exposure and subsequent inhibitor development in children with severe haemophilia A. Haemophilia, 13(2), 149-155.

27. Astermark, J., Berntorp, E., White, G. C., Kroner, B. L., \& MIBS Study Group. (2001). The Malmö International Brother Study (MIBS): further support for genetic predisposition to inhibitor development. Haemophilia, 7(3), 267-272.

28. Van den Berg, H. M., De Groot, P. H. G., \& Fischer, K. (2007). Phenotypic heterogeneity in severe hemophilia. Journal of Thrombosis and Haemostasis, 5, 151-156.

29. Jayandharan, G. R., \& Srivastava, A. (2008, February). The phenotypic heterogeneity of severe hemophilia. In Seminars in thrombosis and hemostasis, 34(1), 128-141. 